\title{
FIXPOINT THEOREM FOR CONTRACTIONS OF A WELL-CHAINED TOPOLOGICAL SPACE ${ }^{1}$
}

\author{
ALLEN S. DAVIS
}

If a map $A: X \rightarrow X: x \rightarrow A x$ in a complete metric space $(X, d)$ is such that, for some $c<1, d(A x, A y) \leqq c d(x, y)$ holds for all $x, y \in X$, then the equation $A x=x$ has in $X$ a unique solution. The usefulness of this "principle of contraction mappings" in analysis has been well illustrated by Kolmogorov and Fomin in [2, pp. 43-51].

Presented with an elegant little theorem of great applicability such as this, one is moved to wonder, how far can it be pushed? Can the concept of "contraction" be made meaningful in more general spaces and, if so, does the quoted fixpoint theorem have a significant generalization? For regular developable spaces an affirmative answer has been provided by Dr. J. Mathews [3], to whom I owe my interest in this question. The present paper offers a fixpoint theorem for well-chained spaces somewhat more general than those furnished with uniformities.

The definitions proposed in the latter half of $\$ 1$ and the lemmata leading to Theorem 2 of $\S 2$ are formulated in as general a manner as seemed compatible with naturalness. It may be that they are of interest independently of the particular fixpoint theorem presented here. (Some of the definitions differ from those tentatively proposed in [1].)

1. Definitions. A family $V_{I}=\left\{V_{\alpha}\right\}_{\alpha \in I}$ of dyadic relations over a set $X$ will be called reflexive if each $V_{\alpha}$ contains the diagonal $\Delta$ of $X \times X$, symmetric if for each $\alpha \in I$ there is a $\beta \in I$ such that (the transpose) $V_{\beta}^{-1} \subseteq V_{\alpha}$, and transitive if for each $\alpha \in I$ there is a $\beta \in I$ and a $\gamma \in I$ such that $V_{\gamma} V_{\beta} \subseteq V_{\alpha}$. (Composition is denoted by juxtaposition and, for powers, by superscripts.) $V_{I}$ is locally transitive if for each $x \in X$, each $\alpha \in I, V_{\gamma} V_{\beta}(x) \subseteq V_{\alpha}(x)$, where $\beta$ and $\gamma$ may depend upon $x$ as well as upon $\alpha$.

If $V_{I}$ is reflexive and closed under the operation of intersection, it will be called a structure for $X$. Thus a reflexive symmetric transitive family of dyadic relations, closed under intersection, is a uniform structure for the set concerned; uniformities are generalized equivalence relations. The structure defined by a metric $d$ for a set $X$ is the

Received by the editors March 23, 1962 and, in revised form, July 23, 1962.

1 The material of this paper is part of the author's doctoral dissertation at the University of Oklahoma. 
family of relations of the form $V_{\alpha}=\{(x, y) \in X \times X ; d(x, y)<\alpha\}$, for $\alpha>0$, and is uniform.

A structure $V_{J}$ for $X$ is as fine as a structure $U_{I}$ for $X$ if for each $\epsilon \in I$ there is a $\delta \in J$ such that $V_{\delta} \subseteq U_{\epsilon}$. Two structures are equivalent if each is as fine as the other. By a space we shall mean a set together with an equivalence class of structures for that set. Notation: $\left(X, V_{I}\right)$, where $V_{I}$ is a representative structure for $X$. A subset of $X$ is open if it contains about each of its points a "sphere" of the form $V_{\alpha}(x)$. Thus topologies are determined by structures. (And vice versa: A topological space is regular if and only if it admits a symmetric locally transitive structure; etc.)

A space $\left(X, V_{I}\right)$ is said to be well-chained from $x \in X$ to $y \in X$ if for each $\alpha \in I$ there is a $k$ such that $y \in V_{\alpha}^{\boldsymbol{k}}(x)$. A well-chained space is one which is well-chained between each pair of its points. $\left(X, V_{I}\right)$ is [sequentially] complete if every Cauchy net [sequence] converges in $X$, i.e., every net [sequence] $x_{D} \subseteq X$ which satisfies: For each $\alpha \in I$ there is an $n \in D$ such that $m \geqq n$ implies $x_{m} \in V_{\alpha}\left(x_{n}\right)$.

Now a map $A: X \rightarrow X: x \rightarrow A x$ is called an $r / s$-map relative to a structure $V_{I}$ for $X, r$ and $s$ being natural numbers, if for every $\alpha \in I$,

$$
V_{\alpha}^{8} \subseteq A^{-1} V_{\alpha}^{r} A,
$$

or equivalently,

$$
A V_{\alpha}^{\boldsymbol{\alpha}} \subseteq V_{\alpha}^{\boldsymbol{r}} A \text {, }
$$

which is to say: if two points of $X$ are no more than an $\alpha$-chain of length $s$ apart, then their images under $A$ will be no more than an $\alpha$-chain of length $r$ apart. If $r<s$, the space "contracts" more or less uniformly under such a map. (But note that these properties are not invariant with respect to equivalence of structures.)

An eventual contraction of a space $\left(X, V_{I}\right)$ is a map $A: X \rightarrow X$ some iteration of which, say $A^{n}$, is an $r / s$-map with $r<s$, relative to some $V_{I}$-equivalent structure for $X$.

These definitions were suggested by the familiar notion of a contraction map in a metric space $(X, d)$. A map $A: X \rightarrow X$ is an eventual metric contraction of $(X, d)$ if, for some metric $d^{*}$ equivalent to $d$, some number $n$, and some real $c<1, d^{*}\left(A^{n} x, A^{n} y\right) \leqq c d^{*}(x, y)$, for all $x, y \in X$. ( $d$ and $d^{*}$ are equivalent if there exist constants $a>0$ and $b$ such that $a d \leqq d^{*} \leqq b d$; equivalent metrics define equivalent structures.)

2. Results. First we note that eventual contractions do indeed generalize the concept of an eventual metric contraction. 
THEOREM 1. Every eventual metric contraction is an eventual contraction of the space whose structure is defined by the given metric.

Proof. Let $A: X \rightarrow X$ be an eventual metric contraction of $(X, d)$ and let $d^{*}$ be the metric required by the above definition. It is not difficult to see that if $n$ is chosen large enough (viz., $\geqq-\log 2 / \log c$ ), then $d^{*}\left(A^{n} x, A^{n} y\right) \leqq \frac{1}{2} d^{*}(x, y)$, for all $x, y \in X$. Given $\alpha>0$, suppose $y \in V_{\alpha}^{2}(x)$, i.e., $d^{*}(x, z)<\alpha$ and $d^{*}(z, y)<\alpha$, for some $z \in X$. Then $d^{*}\left(A^{n} x, A^{n} z\right)<\alpha / 2$ and $d^{*}\left(A^{n} z, A^{n} y\right)<\alpha / 2$, whence $A^{n} y \in V_{\alpha / 2}^{2}\left(A^{n} x\right)$ $\subseteq V_{\alpha}\left(A^{n} x\right)$. Thus $A^{n}$ is a 1/2-map relative to a structure for $X$ equivalent to that defined by $d$, and so $A$ is an eventual contraction of the space.

The converse of Theorem 1 is false, as can be seen by considering the transposition map on a two-point discrete metric space.

The following lemmata lead to a fixpoint theorem.

LEMMA 1. With respect to locally transitive structures, $r / s-m a p s$ are continuous. If either $r=1$ or the structure is transitive, an $r / s-m a p$ is uniformly continuous.

Proof. Straightforward, using $A\left(V_{\beta}(x)\right) \subseteq A V_{\beta}^{s}(x) \subseteq V_{\beta}^{r}(A x)$ $\subseteq V_{\alpha}(A x)$, for $\beta$ properly chosen.

Leмma 2. An r/s-map (relative to some structure) is also an $\mathrm{mr} / \mathrm{ms}$ map relative to the same structure, for every $m=1,2, \cdots$. Likewise, for $s^{\prime} \leqq s$ and $r^{\prime} \geqq r, r / s$-maps are also $r^{\prime} / s^{\prime}$-maps.

Proof. If $V_{\alpha}^{s} \subseteq A^{-1} V_{\alpha}^{r} A$, then $V_{\alpha}^{m s} \subseteq A^{-1} V_{\alpha}^{r} A A^{-1} V_{\alpha}^{r} A \cdots A^{-1} V_{\alpha}^{r} A$ ( $m$ times) $=A^{-1} V_{\alpha}^{m r} A$, since $A A^{-1}=\Delta$. Thus $r / s$-maps are $m r / m s$ maps. The second assertion is also immediate.

REMARK. The "cancellation law" converse of Lemma 2 is not valid in general. But if the structure is uniform, $m r / m s$-maps are $r / s$ maps relative to an equivalent structure.

Lemma 3. The nth iteration of an $r / s-m a p$ is an $r^{n} / s^{n}-m a p$ (re the same structure).

Proof. By induction on $n$. Let $A: X \rightarrow X$ be an $r / s$-map relative to $V_{I}$. Basis: For $n=1$ the assertion holds by hypothesis. Induction Step: Suppose that $A^{n}$ is an $r^{n} / s^{n}$-map. Then $A^{n}$ is also an $s r^{n} / s^{n+1}$ map, by Lemma 2 , and $A$ is also an $r^{n+1} / r^{n} s$-map:

$$
V_{\alpha}^{\alpha^{n+1}} \subseteq A^{-n} V_{\alpha}^{8 r^{n}} A^{n} \subseteq A^{-n-1} V_{\alpha}^{r^{n+1}} A^{n+1},
$$

for each $\alpha \in I$, whence $A^{n+1}$ is an $r^{n+1} / s^{n+1}$-map.

LEMMA 4. If $r<s$ and $A$ is an $r / s$-map relative to a [locally] transitive 
structure $V_{I}$, then there exists a structure equivalent to [defining the same topology as] $V_{I}$ relative to which some iteration of $A$ is a 1/2-map.

Proof. Choose $n$ so that $s^{n} \geqq 2 r^{n}$ and define $U_{I}$ by putting $U_{\alpha}=V_{\alpha}^{r^{n}}$, for each $\alpha \in I$. It is easily seen that $U_{I}$ and $V_{I}$ are equivalent if the latter is transitive (and that they define the same topology if the latter is locally transitive). But for each $\alpha \in I, U_{\alpha}^{2} \subseteq V_{\alpha}^{s^{n}} \subseteq A^{-n} V_{\alpha}^{r^{n}} A^{n}$ $=A^{-n} U_{\alpha} A^{n}$, by Lemma 3 , whence $A^{n}$ is a $1 / 2$-map relative to $U_{I}$.

Lemma 5. Let $A$ be an $r / s$-map of $X$ relative to $V_{I}$. Suppose that, for some $x \in X$, the space is well-chained from $x$ to $A x$. If $r<s$, then for each $\alpha \in I$ there is an $n$ such that, for all $m=1,2, \cdots, A^{m} x \in V_{\alpha}^{n}(x)$.

Proof. By induction on $m$. Let $k$ be such that $A x \in V_{\alpha}^{k}(x)$ and put $n=k s$. Basis: $m=1: A x \in V_{\alpha}^{\boldsymbol{k}}(x) \subseteq V_{\alpha}^{n}(x)$. Induction Step: Suppose $A^{m} x \in V_{\alpha}^{n}(x)$. Then by Lemma 2,

$$
A^{m+1} x \in A V_{\alpha}^{s k}(x) \subseteq A A^{-1} V_{\alpha}^{r k} A(x)=V_{\alpha}^{r k}(A x),
$$

and by definition of $k$ and the fact that $r<s$,

$$
V_{\alpha}^{r k}(A x) \subseteq V_{\alpha}^{r k} V_{\alpha}^{k}(x) \subseteq V_{\alpha}^{s k}(x) .
$$

Thus $A^{m+1} x \in V_{\alpha}^{n}(x)$.

Leмma 6. In a well-chained space, the successive images of a given point under iterations of a 1/2-map form a Cauchy sequence.

Proof. Let $\left(X, V_{I}\right)$ be well-chained and suppose that $A$ is a $1 / 2$ map relative to $V_{I}$. We wish to show that, for every $\alpha \in I$, there is an $n$ such that if $m \geqq n$ then $A^{m} x \in V_{\alpha}\left(A^{n} x\right), x \in X$ being given. Let $N$ be, by Lemma 5 , large enough so that all images of $x$ under iterations of $A$ belong to $V_{\alpha}^{N}(x)$, and choose $n \geqq \log _{2} N$. Then, if $m>n$, we have

$$
A^{m-n} x \in V_{\alpha}^{N}(x) \subseteq V_{\alpha}^{2^{n}}(x) \subseteq A^{-n} V_{\alpha} A^{n}(x),
$$

by Lemma 3 (since $1^{n}=1$ ). That is, $A^{m} x \in A^{n} A^{-n} V_{\alpha} A^{n}(x)=V_{\alpha}\left(A^{n} x\right)$, as desired.

THEOREM 2. Every eventual contraction of a sequentially complete well-chained $T_{0}$-space with transitive structure has a unique fixpoint.

Proof. If $A$ is an eventual contraction of $\left(X, V_{I}\right)$, then some iteration of $A$ is an $r / s$-map, with $r<s$, relative to a structure equivalent to $V_{I}$. Since $V_{I}$ and all equivalent structures are transitive, Lemma 4 tells us that a further iteration of $A$, say $A^{n}=B$, is a $1 / 2$ map relative to a $V_{I}$-equivalent structure, say $U_{J}$. Since the space is 
well-chained, Lemma 6 gives us a Cauchy sequence $x, B x, B^{2} x, \cdots$ $(x \in X)$. But by completeness, this sequence converges to some point $p \in X$. Then, $B$ being continuous (Lemma 1), we have $B p$ $=B \lim _{m \rightarrow \infty} B^{m} x=\lim _{m \rightarrow \infty} B^{m+1} x=p$, so $p$ is a fixpoint for $B$. It is $B$ 's only fixpoint. For suppose that $B q=q$. Since the space is well-chained, for each $\alpha \in J$ there is a $k$ large enough so that $(p, q) \in U_{\alpha}^{2^{k}} \subseteq B^{-k} U_{\alpha} B^{k}$ (using Lemma 3 and remembering that $B$ is a $1 / 2$-map re $U_{J}$ ). But then $(p, q)=\left(B^{k} p, B^{k} q\right) \in U_{\alpha}$. Thus, for every $\alpha \in J, q \in U_{\alpha}(p)$ and, in a like manner, $p \in U_{\alpha}(q)$. Since the space is $T_{0}$, this implies $q=p$. Now we can show that $p$ is a fixpoint for $A$ : Since $B A p=A^{n+1} p=A B p$ $=A p$ and since $B$ has but one fixpoint, $A p=p$. Finally, $p$ is the only fixpoint for $A$ because it is the only one for $B=A^{n}$.

Comment. The example mentioned after Theorem 1 shows that the assumption that the space be well-chained is essential in Theorem 2. It is crucial not only in the proof of existence of a fixpoint but also in the proof of uniqueness (as can be seen by considering the identity map on a discrete space). So Theorem 2 is not quite a true generalization of the fixpoint theorem for metric contractions quoted in the opening paragraph of this paper.

\section{REFERENCES}

1. A. S. Davis, Indexed systems of neighborhoods for general topological spaces, Amer. Math. Monthly 68 (1961), 886-893.

2. A. N. Kolmogorov and S. V. Fomin, Elements of the theory of functions and functional analysis, Vol. I, Graylock Press, Rochester, N. Y., 1957.

3. J. C. Mathews, Fixed point theorem for a contraction mapping in a regular developable space, Doctoral dissertation, Iowa State University, Ames, Iowa, 1959.

UNIVERSITY OF OKLAHOMA 